\title{
Low Surface Brightness Dwarf Galaxies in the Bristol - Anglo-Australian Observatory Virgo Cluster Survey
}

\author{
J. B. Jones, S. Phillipps, J. M. Schwartzenberg \\ Astrophysics Group, Department of Physics, University of Bristol, \\ Tyndall Avenue, Bristol, BS8 1TL, United Kingdom. \\ Q. A. Parker \\ Anglo-Australian Observatory, Siding Spring, Coonabarabran, New \\ South Wales 2357, Australia.
}

\begin{abstract}
We describe a new, deep photographic survey of the Virgo Cluster which uses multiple exposures on Tech Pan film with the United Kingdom Schmidt Telescope to probe the dwarf population to fainter surface brightness limits than previous surveys. We have identified galaxies having sizes ( $\geq 3$ arcsec scale length) and surface brightnesses ( $\leq$ $24.5 \mathrm{R}$ mag $\operatorname{arcsec}^{-2}$ ) characteristic of those expected for dwarf spheroidal galaxies in the cluster. The survey is providing substantial samples of extremely low luminosity galaxies outside the environment of the Local Group and nearby groups for the first time. An initial study of two small areas has found dwarf spheroidal candidates in large numbers $(500$ $\mathrm{deg}^{-2}$ ) which indicate a steep, continuously rising luminosity function at low luminosities.
\end{abstract}

\section{The Virgo Cluster}

The Virgo Cluster, alongside its counterpart in Fornax, is the nearest sizeable galaxy cluster. It is close enough for detailed morphological studies to be possible even for low luminosity dwarf galaxies. It is an irregular, poor cluster of BautzMorgan type III (Abell 1975) and Abell richness class 0. As such, it allows detailed studies of the dwarf population in an environment substantially different from the Local Group and other nearby groups.

A seminal study was carried out by Binggeli, Sandage and Tammann, who generated the Virgo Cluster Catalog consisting of 1277 galaxies classified as certain members and a further 574 possible members over an area of $140 \mathrm{deg}^{2}$ (Binggeli et al. 1985). Membership was assigned by visual inspection, essentially based on the larger angular sizes of the cluster galaxies compared with the background population. Their dwarfs conformed to a moderately steep luminosity function (Sandage et al. 1985, Binggeli et al. 1988). Various detailed studies of cluster members have been performed subsequently, including the dwarf population (e.g. Ferguson \& Sandage 1989, Binggeli \& Cameron 1993, Durrell 1997, Young \& Currie 1998). 
Of particular note, Impey, Bothun \& Malin (1988) performed a survey for large angular size low surface brightness galaxies in a single Schmidt field centred on the M87 cluster core, using a photographic stacking technique. They identified 137 galaxies having central surface brightnesses in the range 23 to $26 \mathrm{~B}$ mag $\operatorname{arcsec}^{-2}$, of which 27 were new detections.

\section{The Bristol - AAO Virgo Cluster Survey}

The properties of the faint end of the galaxy luminosity function are poorly constrained outside the Local Group, both in terms of the numbers and characteristics of the galaxies. Some studies have found evidence for very steep luminosity functions in both cluster (e.g. De Propris et al. 1995, Smith, Driver \& Phillipps 1997, Trentham 1997, 1998a) and field (Loveday 1997, Morgan, Smith \& Phillipps 1998) environments. At the very lowest luminosities, dwarf spheroidal galaxies have been identified in nearby groups (e.g. Caldwell et al. 1998), extending the number of known dSphs and allowing a comparison of their properties with Local Group members. The importance of very low luminosity galaxies in understanding galaxy formation (e.g. Frenk et al. 1996, Kauffmann, Nusser \& Steinmetz 1997) and evolution (Gallagher \& Wyse 1994, Caldwell et al. 1998, Trentham 1998b) demands that progress is made in identifying and studying extremely low luminosity dwarfs in new environments (see Phillipps et al. 1998a, and references therein).

In order to extend surveys of galaxies to fainter surface brightnesses than previous surveys and over a full ten degree square region of the cluster, the Bristol-Anglo-Australian Observatory survey is using multiple exposures with the United Kingdom Schmidt Telescope (UKST) on Kodak Tech Pan emulsion through an R band filter (Schwartzenberg, Phillipps \& Parker 1995, Schwartzenberg \& Phillipps 1995, 1997, Schwartzenberg 1996, Phillipps et al. 1998b, Jones et al. 1998). Six individual exposures of each Virgo field of $1-1 \frac{1}{2}$ hour duration are digitally stacked to give a total integration time of 7 hours. The Tech Pan emulsion combines a high efficiency (approaching 10\%, Parker et al. 1998, Phillipps \& Parker 1993) and fine grains (providing a high imaging resolution of 5 microns and a high uniformity).

The four UKST fields of the Virgo survey area are shown in Figure 1. Field coordinates have been selected so as to cover a $100 \mathrm{deg}^{2}$ region centred on the M87 cluster core ("Cluster A" of Binggeli, Sandage \& Tammann 1987). The data therefore survey both the region of high density in the core and the lower density regions at the periphery. The survey area extends south as far as the M49 cluster ("Cluster B") and includes the "M Cloud" west of the M87 cluster. The central area in the grid has been imaged on all 24 films, in effect providing a further 1 mag depth gain in this region and offering the opportunity of a deeper survey in a restricted area. Table 1 presents details of the four fields.

The photographic study compares very favourably with any CCD surveys currently feasible. The very large solid angles of the Schmidt fields, and the very long integration times, overcome the modest telescope aperture $(1.2 \mathrm{~m})$ and the low quantum efficiency of the emulsion compared with CCDs. It has been possible to perform a very deep survey of a $10 \mathrm{deg} \times 10 \mathrm{deg}$ region in the central part of the Virgo Cluster in the equivalent of only four nights' observing on the 


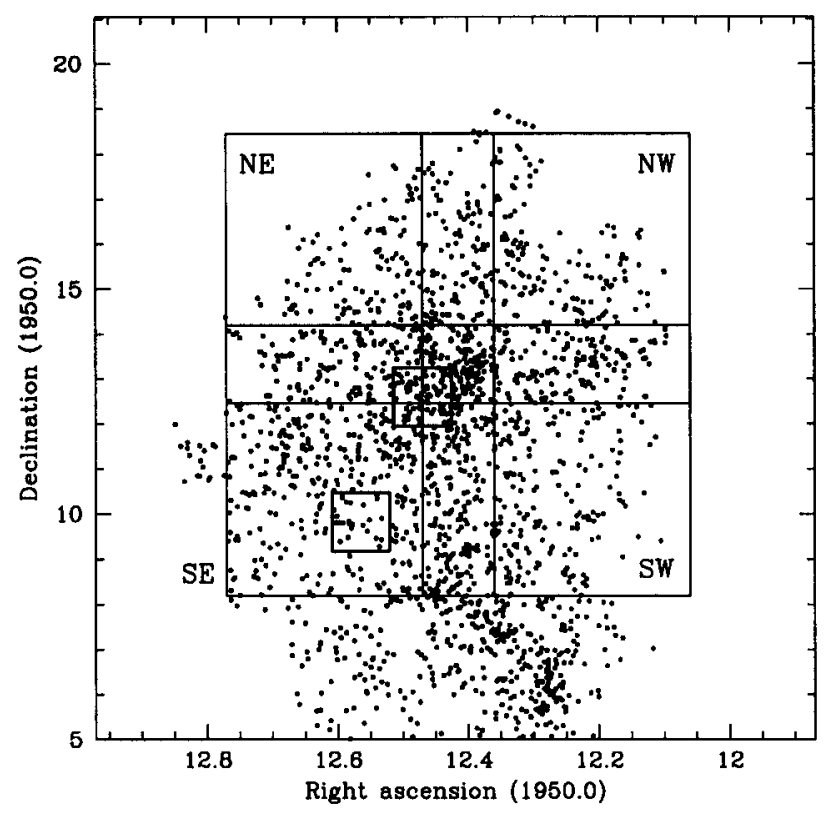

Figure 1. The four UKST fields and the Virgo Cluster. The boundaries of the four Schmidt fields are drawn superimposed on the distribution of cluster members given in the Virgo Cluster Catalog of Binggeli, Sandage \& Tammann (1985). The full $6 \mathrm{deg} \times 6 \mathrm{deg}$ extent of the fields are shown; in practice the scanned regions are slightly smaller. The grid of fields is centred on the M87 core of the cluster. The M49 condensation lies at the southern boundary of the surveyed region. The small squares show the fields used in the initial survey of Section 5 .

Schmidt. An equivalent survey to the same depth using CCDs might reasonably take several weeks, dependent on the details of detector and telescope. To date, the photographic observations are $96 \%$ complete.

\section{Data Reduction and Analysis}

The photographic data are digitised using the SuperCOSMOS plate measuring machine at the Royal Observatory Edinburgh. Only the best quality films are used for stacking: adding poor quality films does not provide a useful gain in depth (Bland-Hawthorn, Shopbell \& Malin, 1993). With 10 micron wide pixels, corresponding to 0.67 arcsec on the sky, data extents are very large, causing particular data processing difficulties. 
Table 1. The four quadrants of the surveyed area.

\begin{tabular}{|c|c|c|c|c|}
\hline \multirow[t]{2}{*}{ Field } & \multicolumn{2}{|c|}{ Central coordinates } & \multirow{2}{*}{$\begin{array}{l}\text { No. exposures } \\
\text { used }\end{array}$} & \multirow{2}{*}{$\begin{array}{l}\text { Total integ } \\
\text { time }\end{array}$} \\
\hline & R.A. (1950.0) & Dec. $(1950.0)$ & & \\
\hline Northwest & $12^{\mathrm{h}} 16^{\mathrm{m}}$ & $+15.4^{\circ}$ & 6 & $6.3 \mathrm{hr}$ \\
\hline Southwest & $12 \quad 16$ & +11.2 & 6 & 6.0 \\
\hline Northeast & 12 & +15.4 & 6 & $6.3^{1}$ \\
\hline Southeast & 34 & +11.2 & 6 & 7.1 \\
\hline
\end{tabular}

The individual exposures are sky-subtracted using a pixel-by-pixel subtraction of a median-filtered version of the data. The use of a 3 arcmin square spatial filter maximises the ability to detect small, faint images but limits the survey, at least at the present time, to small galaxy images - for example, the larger galaxies in the survey of Impey, Bothun and Malin (1988) would be partially removed. All six films of a given part of sky have their intensities normalised to the same scale, thus correcting for factors such as differences in exposure time, atmospheric transmission or the details of the development process. Coaddition of the six exposures is accomplished using median stacking, which efficiently overcomes problems such as film defects, dust particle images or satellite trails that affect single films. Image detection is performed with a connected-pixel algorithm using a detection threshold $\mu_{l i m}=25.5 \mathrm{R} \mathrm{mag} \mathrm{arcsec}{ }^{-2}$ and a minimum image area above this isophote of $A_{l i m}=11 \operatorname{arcsec}^{2}$. These parameters ensure that each detection will have a signal-to-noise ratio of least 10 and magnitudes $R \leq 22$ mag.

\section{Identifying Virgo Dwarf Spheroidals}

Whilst the detection of galaxies in the direction of the Virgo Cluster may not present a particular challenge in itself, the identification of cluster members is significantly more difficult. Nearby clusters cover large angular scales and consequently the cluster population is swamped by the numerically dominant background population (see, for example, the review by Trentham 1998b). In the absence of redshift information (for example because of the practicalities of performing spectroscopy for very large samples of galaxies extending over wide areas, or because of the difficulties of obtaining spectra for low surface brightness galaxies), membership for the dwarf population must often be assigned on morphological grounds. Binggeli, Sandage \& Tammann (1985) were able to assign membership for dwarf galaxies in the cluster on the basis of the galaxies' visual appearance: the dwarfs are characterised by their low surface brightnesses for their sizes.

In the present survey, even though the galaxies are more extreme than those of the Virgo Cluster Catalog, it is still possible to isolate samples of cluster 
galaxies likely to suffer only a small degree of contamination by the background population. A comparison of Virgo photographic data with deep CCD data from a South Galactic Pole field shows that the background contamination is as small as several percent for galaxies having central surface brightnesses in the range $\mu_{0}=22.0$ to $24.5 \mathrm{R} \mathrm{mag} \operatorname{arcsec}^{-2}$ and exponential scale lengths $a \geq 3$ arcsec, even when allowance is made for differences in resolution between the two data sets. These issues are discussed in greater detail by Schwartzenberg (1996) and by Jones et al. (1998).

The galaxies selected have properties comparable to those that Local Group dwarf spheroidals would have if they were at the distance of the Virgo Cluster. They have exponential scale lengths $h \geq 260 \mathrm{pc}$ and absolute magnitudes $M_{\mathrm{R}}=$ -11 to -16 (for an assumed distance modulus of $31.3 \mathrm{mag}$, equivalent to $H_{0}=$ $70 \mathrm{~km} \mathrm{~s}^{-1} \mathrm{Mpc}^{-1}$ ). Local Group dwarf spheroidals have sizes between $h=90$ and $400 \mathrm{pc}$ and absolute magnitudes $M_{\mathrm{R}}=-9$ to -14 . The Virgo objects generally have fainter surface brightnesses than the galaxies of Binggeli, Sandage and Tammann (1985) and are smaller than those of Impey, Bothun and Malin (1988).

\section{The Initial Survey}

A preliminary study of two subfields in the Southeast quadrant of the full survey area has been carried out. The SuperCOSMOS scans of the quadrant were provided as nine sections, each $7680 \times 7680$ pixels in size, two of which were used for the initial survey (Schwartzenberg et al., 1995). Excluding their edges, both fields are $1.3 \mathrm{deg}$ square, providing a total area of $3.2 \mathrm{deg}^{2}$. The fields, listed in Table 5., were selected to sample the core of the cluster and a region of lower density $3.1 \mathrm{deg}$ to the southeast. The core field included M87, although due to the raised background light levels, the region immediately around M87 was excluded from the study. The data were further subdivided into $2280 \times 2280$ pixel subregions for data processing because of computer hardware limitations. Fuller details are given by Schwartzenberg (1996) and Phillipps et al. (1998b).

Table 2. Details of the fields studied in the initial survey.

\begin{tabular}{lcc}
\multicolumn{1}{c}{ Property } & Core field & Outer field \\
\hline Field area & $1.58 \mathrm{deg}^{2}$ & $1.61 \mathrm{deg}^{2}$ \\
Field centre: & & \\
R.A.(1950) & $12^{\mathrm{h}} 28.2^{\mathrm{m}}$ & $12^{\mathrm{h}} 33.9^{\mathrm{m}}$ \\
Dec.(1950) & $+12^{\circ} 36^{\prime}$ & $+09^{\circ} 49^{\prime}$
\end{tabular}

A total of 56000 images were detected over the two fields. From these a subsample of galaxies was selected having central surface brightnesses in the range $\mu_{0}=22.0$ to $24.5 \mathrm{R}$ mag $\operatorname{arcsec}^{-2}$ and scale lengths $a \geq 3$ arcsec after fitting exponential light profiles to the data. Through a detailed comparison of the numbers of Virgo galaxies with the South Galactic Pole field population at 

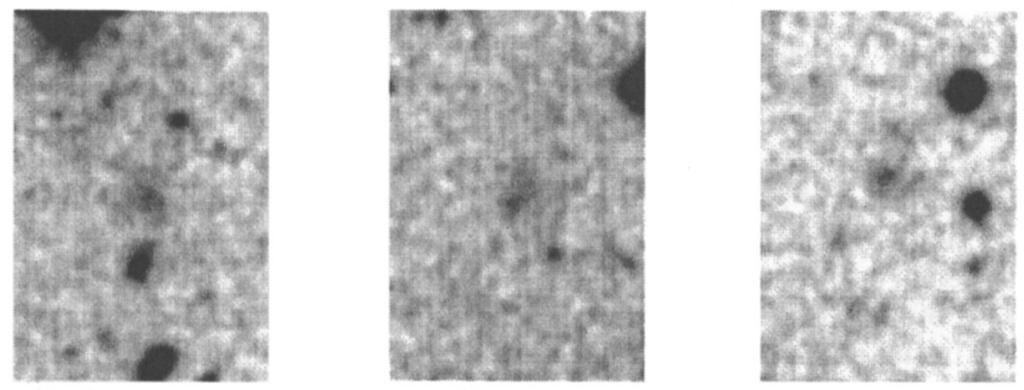

Figure 2. Examples of candidate Virgo Cluster dwarf spheroidal galaxies from the initial survey of $3.2 \mathrm{deg}^{2}$. The images have been produced by coadding six UKST exposures on Tech Pan film. None of the galaxies appears in either the Virgo Cluster Catalog or the sample of Impey, Bothun \& Malin (1988). Each frame is 1.0 arcmin in width. North is at the top.

each point in the magnitude - surface brightness plane, it is possible to remove the background contamination. The overall contamination of the sample is expected to be 8 per cent. After this background subtraction, the sample contains 1570 galaxies across the two fields. A luminosity function can be constructed by binning the background-subtracted galaxy densities by magnitude. The luminosity function of this sample is found to be steep, with a formal faint end slope of $\alpha=-2.2$ (Phillipps et al. 1998b), and as such is comparable with the steep functions found in some more distant clusters (e.g. Driver et al. 1994, De Propris et al. 1995, Smith, Driver \& Phillipps 1997, Wilson et al. 1997, Trentham 1997, 1998a). At the bright end, galaxy numbers are consistent with those of Sandage, Binggeli and Tammann (1985). The dwarf density in the cluster core field is actually smaller than that in the outer field ( 430 galaxies $\mathrm{deg}^{-2}$ against $560 \mathrm{deg}^{-2}$ ). The dense environment in the cluster core, and particularly the presence of M87, may have a direct effect on the low surface brightness galaxy population, either through the removal of these galaxies or suppressing their formation. A similar effect has been found in the core of the Coma Cluster by Thompson \& Gregory (1993).

\section{The Full Cluster Survey}

Progress is underway on reducing the full $100 \mathrm{deg}^{2}$ Virgo survey area. To date, 23 of the required 24 exposures have been taken. It is intended that the full survey will measure the background population using data identical in format to those from Virgo, reducing systematic errors which might be introduced during the subtraction of the background galaxy numbers. Work is therefore progress- 
ing on the Virgo Northwest field and on a background field centred at right ascension $10 \mathrm{~h} 40 \mathrm{~m}$, declination $0^{\circ} 00^{\prime}(1950)$. The photometric calibration has been accomplished using $R$ band observations on the Anglo-Australian Telescope obtained for other projects.

For data reduction, each field is subdivided into 16 subregions, each $6800 \times$ 6800 pixels. Data are stored as 4-byte numbers per pixel, and therefore each $1 / 16$-th section is 185 Mbyte in size. New computer hardware resources provide sufficient memory to reduce each $6800 \times 6800$ pixel section as a whole, avoiding any need to break these sections into a large number of smaller regions as was done in the initial survey. This has the advantage of much simpler data handling than would be needed if large numbers of small sections were used. Similarly, establishing astrometric reference frames for a very large number of different sections would be prohibitive.

As in the case of the initial survey of $3.2 \mathrm{deg}^{2}$, emphasis is being put on searches for small angular size low surface brightness dwarfs. However, the feasibility of performing a parallel survey with a different sky background subtraction is being investigated, in order to allow searches for large angular size low surface brightness galaxies of the type studied by Impey, Bothun and Malin (1988), but over a wider area than was available for their study.

\section{Conclusions}

The new survey is already providing large samples of candidate Virgo Cluster dwarf spheroidal galaxies suitable for more detailed study. The galaxies have absolute magnitudes as faint as $M_{\mathrm{R}}=-11$ to -16 (for $H_{0}=70 \mathrm{~km} \mathrm{~s}^{-1} \mathrm{Mpc}^{-1}$ ). They will be important for addressing questions relating to the properties and evolution of the lowest luminosity galaxies in an environment very different from that in nearby galaxy groups. An initial survey of $3.2 \mathrm{deg}^{2}$ has found a high density of these galaxies and evidence for a steep luminosity function. The survey is continuing over an area of $100 \mathrm{deg}^{2}$. The very large numbers of dwarf spheroidals should reduce the errors in the faint end of the luminosity function and help to define its shape. They will enable clear differences to be found at these very faint limits between the cluster core and periphery to significantly better accuracy than has been possible for any other cluster before now. A parallel survey of the Fornax Cluster is planned for which several films are already available.

Acknowledgments. We wish to thank the UKST and SuperCOSMOS staff in providing their usual excellent service.

\section{References}

Abell, G. O. 1975, in 'Galaxies and the Universe', A. Sandage, M. Sandage \& J. Kristian, University of Chicago Press, p. 601

Binggeli, B., \& Cameron, L. M. 1993, A\&AS, 98, 297

Binggeli, B., Sandage, A., \& Tammann, G. A. 1985, AJ, 90, 1681

Binggeli, B., Sandage, A., \& Tammann, G. A. 1988, ARA\&A, 26, 509 
Binggeli, B., Tammann, G. A., \& Sandage, A. 1987, AJ, 94, 251

Bland-Hawthorn, J., Shopbell, P. L., \& Malin, D. F. 1993, AJ, 106, 2154

Caldwell, N., Armandroff, T. E., Da Costa, G. S., \& Seitzer, P. 1998, AJ, 115, 535

De Propris, R., Pritchett, C. J., Harris, W. E., \& McClure, R. D. 1995, ApJ, 450,534

Durrell, P. R. 1997, AJ, 113, 531

Frenk, C. S., Evrard, A. E., White, S. D. M., \& Summers, F. J. 1996, ApJ, 472, 460

Ferguson, H. C., \& Sandage, A. 1989, ApJ, 346, L53

Gallagher, J. S., Wyse, R. F. G. 1994, PASP, 106, 1225

Impey, C. D., Bothun, G. D., \& Malin, D. F. 1988, ApJ, 330, 634

Jones, J. B., Phillipps, S., Schwartzenberg, J. M., \& Parker, Q. A. 1998, in 'Dwarf Galaxies and Cosmology', T. X. Thuân et al., Gif-sur-Yvette: Editions Frontières, in press. astro-ph/9805287

Kauffmann, G., Nusser, A., \& Steinmetz, M. 1997, MNRAS, 286, 795

Loveday, J. 1997, ApJ, 489, 29

Morgan, I., Smith, R. M., \& Phillipps, S. 1997, MNRAS, 295, 99

Parker, Q. A., Malin, D. F., Canon, R. D., Phillipps, S., \& Russell, K. S. 1998, MNRAS, submitted

Phillipps, S., Driver, S. P., Couch, W. J., \& Smith, R. M. 1998a, ApJ, 498, L119

Phillipps, S., \& Parker, Q. A. 1993, MNRAS, 265, 385

Phillipps, S., Parker, Q. A., Schwartzenberg, J. M., \& Jones, J. B. 1998b, ApJ, 493, L59

Sandage, A., Binggeli, B., \& Tammann, G. A. 1985, AJ, 90, 1759

Schwartzenberg, J. M., 1996, Ph.D. Thesis, University of Bristol

Schwartzenberg, J. M., \& Phillipps, S. 1995, Anglo-Australian Observatory Newsletter, 72,6

Schwartzenberg, J. M., \& Phillipps, S. 1997, Astroph. Lett. \& Comm., 36, 279

Schwartzenberg, J. M., Phillipps, S., \& Parker, Q. A. 1995, A\&A, 293, 332

Schwartzenberg, J. M., Phillipps, S., \& Parker, Q. A. 1996, A\&AS, 117, 179

Smith, R. M., Driver, S. P., \& Phillipps, S. 1997, MNRAS, 287, 415

Thompson, L. A., \& Gregory, S. A. 1993. AJ, 106, 2197

Trentham, N. 1997, MNRAS, 286, 133

Trentham, N. 1998a, MNRAS, 293, 71

Trentham, N. 1998b, in 'Dwarf Galaxies and Cosmology', T. X. Thuân et al., Gif-sur-Yvette: Editions Frontières, in press. astro-ph/9804013

Wilson, G., Smail, I., Ellis, R. S., \& Couch, W. J. 1997, MNRAS, 284, 915

Young, C. K., \& Currie, M. J. 1998, A\&AS, 127, 367 